\title{
Role of fasting duration and weekday in incretin and glucose regulation
}

\author{
Kim K B Clemmensen ${ }^{1}$, Jonas S Quist ${ }^{1}$, Dorte Vistisen ${ }^{1}$, Daniel R Witte ${ }^{2,3}$, Anna Jonsson ${ }^{4}$, Oluf Pedersen ${ }^{4}$, \\ Torben Hansen ${ }^{4}$, Jens J Holst ${ }^{4,5}$, Torsten Lauritzen ${ }^{6}$, Marit E Jørgensen ${ }^{1,7}$, Signe Torekov ${ }^{4,5}$ and Kristine Færch ${ }^{1}$ \\ 'Department of Clinical Epidemiology, Steno Diabetes Center Copenhagen, Gentofte, Denmark \\ ${ }^{2}$ Department of Public Health, Aarhus University, Aarhus, Denmark \\ ${ }^{3}$ Danish Diabetes Academy, Odense, Denmark \\ ${ }^{4}$ NNF Center for Basic Metabolic Research, University of Copenhagen, Copenhagen, Denmark \\ ${ }^{5}$ Department of Biomedical Sciences, University of Copenhagen, Copenhagen, Denmark \\ ${ }^{6}$ Section for General Practice, Department of Public Health, Aarhus University, Aarhus, Denmark \\ ${ }^{7}$ National Institute of Public Health, University of Southern Denmark, Copenhagen, Denmark
}

Correspondence should be addressed to K K Clemmensen: kim.katrine.bjerring.clemmensen.01@regionh.dk

\begin{abstract}
Fasting duration has been associated with lower fasting blood glucose levels, but higher 2-h post-load levels, and research has indicated an adverse effect of 'weekend behavior' on human metabolism. We investigated associations of fasting duration and weekday of examination with glucose, insulin, glucagon and incretin responses to an oral glucose tolerance test (OGTT). This cross-sectional study is based on data from the ADDITION-PRO study, where 2082 individuals attended a health examination including an OGTT. Linear regression analysis was applied to study the associations of overnight fasting duration and day of the week with glucose, insulin, glucagon, glucose-dependent insulinotropic polypeptide (GIP) and glucagon-like peptide 1 (GLP-1) responses to an OGTT. We found that a $1 \mathrm{~h}$ longer fasting duration was associated with $1.7 \%(95 \% \mathrm{Cl}: 0.8,2.5)$ higher 2 -h glucose levels, as well as a 3.0\% (95\% Cl: 1.3,4.7) higher GIP and 2.3\% (95\% Cl: 0.3,4.4) higher GLP-1 response. Fasting insulin levels were $20.6 \%$ (95\% Cl: 11.2,30.7) higher on Mondays compared to the other weekdays, with similar fasting glucose levels $(1.7 \%, 95 \% \mathrm{Cl}: 0.0,3.4)$. In this study, longer overnight fasting duration was associated with a worsening of glucose tolerance and increased incretin response to oral glucose. We found higher fasting insulin levels on Mondays compared to the other days of the week, potentially indicating a worsened glucose regulation after the weekend.
\end{abstract}

\section{Key Words}

- fasting duration
- incretin

- oral glucose tolerance test

- weekday

- metabolism

\section{Introduction}

Glucose metabolism exhibits circadian rhythmicity $(1,2)$. Longer fasting duration before an oral glucose tolerance test (OGTT) has been associated with lower fasting glucose but higher post-load glucose levels (3). Additionally, the time of the day of an OGTT seems to influence the glucose response with higher post-load blood glucose levels in the afternoon and evening compared to the morning $(1,3)$. The incretin hormones glucagon-like peptide 1 (GLP-1) and glucose-dependent insulinotropic polypeptide (GIP) account for a substantial part of the insulin secreted after ingestion of glucose $(4,5,6)$. It has been shown that the incretin effect is impaired in type 2 diabetes and obesity $(5,7,8)$. Also, the secretion of both GLP-1 and GIP has been shown to exhibit a circadian pattern, with lower levels in the morning and higher levels in the late afternoon $(9,10)$.

The timing of sleep and meals shows variation over the week, with other activity patterns in the weekend
Endocrine Connections (2020) 9, 279-288 
compared to the weekdays, a term called social jetlag (11, 12). A large observational study has reported higher energy intake and poorer diet quality in weekends compared to weekdays (13). Also, studies on the effect of weekday on triglyceride levels showed higher triglyceride levels on Mondays compared to Fridays $(14,15)$, indicating an acute adverse effect of 'weekend behavior' on metabolism. 'Weekend behavior' is the combination of changes in dietary intake as well as a shift in sleep schedule in the weekends compared to the daily rhythm in the weekdays $(11,12,13)$. However, knowledge on the role of weekend behavior on glucose metabolism and incretin hormone response is lacking.

The objectives of the present cross-sectional study were to investigate associations of fasting duration and weekday of examination with glucose, insulin, glucagon, GIP and GLP-1 responses to an OGTT. We hypothesized that longer overnight fasting duration was associated with greater incretin response and lower fasting levels of glucose and insulin. Moreover, we anticipated that glucose regulation on Mondays would be worse than on the other weekdays because of less healthy 'weekend behavior'.

\section{Materials and methods}

This cross-sectional study was based on data from the Danish ADDITION-PRO study. The ADDITION-PRO study is nested in the ADDITION-Denmark study, a longitudinal risk-stratified cohort study of individuals at high risk of type 2 diabetes conducted from 2001 to 2006 (16). A subset of the participants from the ADDITION-Denmark study was invited to a follow-up health examination conducted from 2009 to 2011. The subset included both participants with diabetes, impaired glucose tolerance and participants at low to high risk of developing type 2 diabetes assessed by glycaemic status and score on a slightly modified version of the Danish diabetes risk questionnaire $(16,17)$. Of the 4188 individuals invited, 2082 (50\%) attended the follow-up examination. The ADDITION-PRO study is described in detail elsewhere (16). Of the 2082 participants in the ADDITION-PRO study, we excluded 336 with known diabetes and 20 who had fasted for less than $8 \mathrm{~h}$ (minimum required fasting duration), leaving 1726 participants for analysis.

The ADDITION-PRO study was conducted in accordance with the Helsinki Declaration, and participants gave written informed consent before testing. The study was approved by the Ethical Committee of the Central Denmark Region (No. 20080229).

\section{Biochemical measurements}

Blood samples for the measurements of glucose, insulin, glucagon, GIP and GLP-1 were drawn in the fasting state and 30- and 120-min post-load during a standard $75 \mathrm{~g}$ OGTT. The participants were asked to fast for a minimum of $8 \mathrm{~h}$ prior to the test. The timing of their last meal before the test was recorded as well as the date and time of the test. All OGTTs were performed on weekdays (MondayFriday), with 199 participants on Mondays, 914 on Tuesdays, 126 on Wednesdays, 318 on Thursdays and 166 on Fridays. Three participants had missing information on weekday of examination and were omitted from the weekday analyses.

Glucose and insulin were analysed in the central laboratory at the Steno Diabetes Center Copenhagen, Gentofte, Denmark. For the glucose measurements, the plasma was prepared immediately after collection in fluoride-heparin coated tubes, placed on ice and thereafter centrifuged at $2200 \mathrm{~g}$ for $10 \mathrm{~min}$ at $4^{\circ} \mathrm{C}$ (16). From 2009 to 2010, glucose was measured using the Hitachi 912 system (Roche Diagnostics), but gradually in 2010 this was changed to the Vitros 5600 Integrated System (Ortho Clinical Diagnostics, Illkirch Cedex, France) (16). For the insulin measurements, whole blood was incubated for $0.5-1.5 \mathrm{~h}$ at room temperature, centrifuged for 10 min at $2200 \boldsymbol{g}$ and serum samples were analysed with an immunoassay (AutoDELFIA, Perkin Elmer) (16). The intraassay coefficient of variation was 0.011 , and the interassay coefficient of variation was 0.036 with serum insulin levels of $129 \mathrm{pmol} / \mathrm{L}$ (18).

Samples for measurement of glucagon, GIP and GLP-1 were first stored in a biobank and were after completion of the study analysed within 2 months using identical quality controls and batches for all reagents $(16,19)$. Plasma stored in the biobank was from blood sampled in chilled EDTA coated tubes, put on ice immediately and centrifuged within $30 \mathrm{~min}$ after collection at $2200 \boldsymbol{g}$ for $10 \mathrm{~min}$ at $4^{\circ} \mathrm{C}$ and stored at $-80^{\circ} \mathrm{C}$ (16). Glucagon was measured using radioimmuno assays using a $\mathrm{COOH}$ terminus which measures intact glucagon; for details see Færch et al. (19). Total plasma GIP and GLP-1 concentrations (comprising both intact GIP and GLP-1 and the metabolites GIP(3-42) and GLP-1(9-36)amide, respectively) were measured by radioimmuno assays as previously described (19). The analytical detection limit for GIP and glucagon was $1 \mathrm{pmol} / \mathrm{L}$, and the intraassay coefficient of variation was $6.0 \%$ and the interassay coefficient of variation was $15 \%(19,20)$. The analytical detection limit for GLP-1 was $1 \mathrm{pmol} / \mathrm{L}$, the intraassay

This work is licensed under a Creative Commons Attribution-NonCommercial-NoDerivatives 4.0 Internationab ticense.ifica.com at 04/26/2023 12:15:05PM 
coefficient of variation was $6.0 \%$ and the interassay coefficient of variation was $1.5 \%$ (19).

\section{Calculations}

Early and total responses to the OGTT in plasma glucose, serum insulin, plasma glucagon, plasma GIP and plasma GLP-1 were calculated as total areas under the curves (tAUCs) using the trapezoid rule from the basal state to $30 \mathrm{~min}$ (early response, $\mathrm{tAUC}_{0-30}$ ) and $120 \mathrm{~min}$ (total response, $\left.\mathrm{tAUC}_{0-120}\right)$. Relative early response $\left(\mathrm{rAUC}_{0-30}\right.$ ) was calculated as the ratio $\mathrm{tAUC}_{0-30} /$ (fasting concentration $\times$ $30 \mathrm{~min}$ ) and relative total response $\left(\mathrm{rAUC}_{0-120}\right)$ as tAUC $_{0-120} /$ (fasting concentration $\times 120 \mathrm{~min}$ ). Thus, the rAUC reflects the change in the concentrations of glucose and the different hormones relative to the basal (fasting) level. The rAUC is always positive and can therefore be logarithmically transformed.

The overnight fasting duration was calculated as the time between the last meal and the start of the OGTT. Weekdays were grouped into Monday (yes/no). Smoking status was dichotomized as current smoker vs non-smoker. Physical activity energy expenditure was calculated from combined accelerometry and heart rate monitoring for 7 days. A step test was used to assess cardiorespiratory fitness (16).

\section{Statistical analysis}

Differences in characteristics between day of examination were compared with chi-square or $t$-test. Linear regression analysis was used to study the associations of fasting duration and weekday of examination with plasma levels at 0,30 and $120 \mathrm{~min}$ as well as with early and total relative responses of glucose, insulin, glucagon, GIP and GLP-1. The analyses were adjusted for age, sex, BMI and smoking status as well as weekday or fasting duration, respectively. The outcome variables were logarithmically transformed before analysis to obtain normally distributed model residuals.

$\mathrm{R}$ version 3.6.0 was used for the statistical analyses. A two-sided a-level of 0.05 was used.

\section{Results}

\section{Participant characteristics}

Participant characteristics, stratified by weekday of examination, are presented in Table 1.
Participants examined on Mondays were slightly healthier than those examined on the other weekdays with lower age and $\mathrm{HbA}_{1 \mathrm{c}}$ levels as well as lower BMI and waist circumference. The OGTT started between 07:00 and 10:00 $\mathrm{h}$ in the morning for more than 99\% of the participants, with a median (quartile 1, quartile 3 ) starting time at 08:25 h (07:50 h,08:51 h).

There was a slightly higher proportion of men among those who attended (53\%) than among non-attendees (43\%; $P<0.001)$. Sixty-eight percent of attendees had no relatives with diabetes compared to $74 \%$ of the nonattendees $(P<0.001)$, and a lower proportion of the attendees than the non-attendees were normal weight (36\% vs 40\%; $P=0.006$ ). There were no differences in age, hypertension and physical inactivity between attendees and non-attendees (16).

\section{Fasting duration}

The crude and adjusted estimates from the linear regression analyses of overnight fasting duration with glucose and hormone levels are shown in Table 2. In the adjusted analyses, fasting glucose levels were not associated with the length of the overnight fast (Table 2, Fig. 1). However, a $1 \mathrm{~h}$ longer fasting duration was associated with $1.7 \%$ (95\% CI: 0.8,2.5) higher 2-h glucose levels and 3.6\% (95\% CI: 1.4,5.8) higher 2-h insulin levels (Table 2). No association between any of the glucagon measures and fasting duration was observed (Fig. 1 and Table 2).

With each additional hour of overnight fast, the mean fasting levels of GIP were 2.4\% (95\% CI: -4.1,-0.7) lower and fasting GLP-1 levels were 2.9\% (95\% CI: -5.1,-0.6) lower (Fig. 2 and Table 2). Early GIP ( rAUC $_{0-30 \mathrm{~min}}$ ) and total (rAUC ${ }_{0-120 \text { min }}$ ) GIP and GLP-1 responses were positively associated with fasting duration (Fig. 2 and Table 2).

\section{Weekdays}

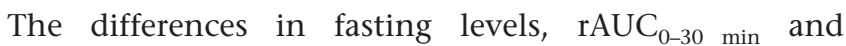
$\mathrm{rAUC}_{0-120 \mathrm{~min}}$ for glucose, insulin, glucagon, GIP and GLP-1 between Mondays and the other days of the week combined are shown in Table 3. Fasting glucose levels were almost similar on Mondays compared to the other days of the week but the $\mathrm{rAUC}_{0-30 \text { min }}$ and $\mathrm{rAUC}_{0-120 \text { min }}$ for glucose were lower on Mondays (Table 3). Fasting insulin levels were $20.6 \%$ (95\% CI: 11.2,30.7) higher and 30-min insulin levels were 13.1\% (95\% CI: 2.8,24.4) higher on Mondays. $\mathrm{rAUC}_{0-120 \mathrm{~min}}$ of insulin was lower $(-8.4 \%$, 95\% CI: $-14.7,-1.5, P=0.017)$ on Mondays compared with the other week days (Table 3 ). The measures for glucagon, 
Table 1 Characteristics of the ADDITION-PRO study population by weekday of examination.

\begin{tabular}{|c|}
\hline$n$ \\
\hline $\operatorname{Men}(n(\%))$ \\
\hline Age (years) \\
\hline Height $(\mathrm{cm})$ \\
\hline $\mathrm{BMI}\left(\mathrm{kg} / \mathrm{m}^{2}\right)$ \\
\hline Waist circumference (cm) \\
\hline Hip circumference (cm) \\
\hline Fat percentage \\
\hline $\mathrm{HbA}_{1 \mathrm{c}}(\%)$ \\
\hline $\mathrm{HbA}_{1 \mathrm{c}}(\mathrm{mmol} / \mathrm{mol})$ \\
\hline Glucose tolerance status ( $n(\%))$ \\
\hline i-IFG \\
\hline $\mathrm{i}-\mathrm{IGT}$ \\
\hline IFG\&IGT \\
\hline Screen detected type 2 diabetes \\
\hline Overnight fasting (h) \\
\hline Time of fasting sample (h:min) \\
\hline PAEE (kJ/kg/day) \\
\hline $\mathrm{CRF}(\mathrm{mL} \mathrm{O} / \mathrm{kg} / \mathrm{min})$ \\
\hline Fasting plasma glucose $(\mathrm{mmol} / \mathrm{L})$ \\
\hline Fasting plasma insulin (pmol/L) \\
\hline Fasting plasma glucagon (pmol/L) \\
\hline Fasting plasma GLP-1 (pmol/L) \\
\hline Fasting plasma GIP (pmol/L) \\
\hline
\end{tabular}

\begin{tabular}{c}
\hline Monday \\
\hline 199 \\
$100(50)$ \\
$64(7)$ \\
$170(9)$ \\
$26.5(4.1)$ \\
$94.2(13.1)$ \\
$100.6(8.2)$ \\
$32.0(7.6)$ \\
$5.6(0.4)$ \\
$38(5)$ \\
$41(20.6)$ \\
$17(8.5)$ \\
$19(9.5)$ \\
$22(11.1)$ \\
$11.8(10.6,12.9)$ \\
$08: 06(07: 42,08: 48)$ \\
$34.3(15.7)$ \\
$30.1(5.3)$ \\
$6.1(0.7)$ \\
$52.2(47.3)$ \\
$11.8(8.0)$ \\
$11.8(6.8)$ \\
$9.7(5.9)$
\end{tabular}

\begin{tabular}{c}
\hline Tuesday-Friday \\
\hline 1524 \\
$821(54)$ \\
$66(7)$ \\
$170(9)$ \\
$27.3(4.6)$ \\
$95.5(13.0)$ \\
$101.7(9.9)$ \\
$32.0(8.4)$ \\
$5.7(0.4)$ \\
$39(5)$ \\
$293(19.2)$ \\
$117(7.7)$ \\
$143(9.4)$ \\
$161(10.6)$ \\
$11.5(10.3,13.0)$ \\
$08: 24(08: 00,08: 54)$ \\
$32.3(15.8)$ \\
$29.9(5.4)$ \\
$6.0(0.8)$ \\
$45.4(32.5)$ \\
$11.3(8.1)$ \\
$12.1(5.9)$ \\
$9.7(6.1)$
\end{tabular}

\begin{tabular}{r}
\hline P-value \\
\hline 0.375 \\
$<0.001$ \\
0.945 \\
0.020 \\
0.200 \\
0.132 \\
0.988 \\
0.004 \\
0.004 \\
0.944 \\
- \\
- \\
- \\
- \\
0.450 \\
$<0.001$ \\
0.203 \\
0.589 \\
0.395 \\
0.009 \\
0.394 \\
0.479 \\
0.952
\end{tabular}

Data are shown as means (s.D.) or median (quartile 1, quartile 3).

CRF, cardiorespiratory fitness; GIP, glucose-dependent insulinotropic polypeptide; GLP-1, glucagon-like peptide 1; IFG, impaired fasting glucose; i-IFG, isolated IFG; IGT, impaired glucose tolerance; i-IGT, isolated IGT; PAEE, physical activity energy expenditure.

GIP and GLP-1 did not differ between Mondays and the other weekdays.

\section{Discussion}

We found slightly higher 2-h glucose and insulin levels in participants who had a longer overnight fasting duration prior to their OGTT. For GIP and GLP-1, 2-3\% higher response to oral glucose $\left(\mathrm{rAUC}_{0-120}\right)$ with $1 \mathrm{~h}$ longer overnight fasting durations were observed. We found similar levels of fasting glucose on Mondays compared to the other days of the week combined, while fasting insulin was found to be $20.6 \%$ higher on Mondays, indicating a worsened glucose regulation after the weekend.

\section{Comparison with other studies}

Our findings partly support previous findings from the large British population-based Whitehall II cohort study (3), where longer fasting duration was associated with higher 2-h post-load glucose levels. That longer fasting duration is associated with higher 2 -h post-glucose load circulating glucose and insulin concentrations is not surprising, as the body with prolonged fasting can be expected to become more insulin resistant (21). However, we did not find an association between fasting duration and fasting glucose levels as was found in the Whitehall II study (3). One possible explanation for the discrepancy is that the Whitehall II study included the time of day of the OGTT in their analysis, which attenuated the association of fasting duration with fasting glucose levels (3). In the present study, the time range of the starting time of the OGTT was too short to add information to the analysis. In a large primary care-based study of more than 25,000 individuals, fasting glucose levels were stable when the fasting duration exceeded approximately $3 \mathrm{~h}$ (22), which is in line with our results. Contrary to our findings, a study including 1006 women found 4\% lower 2-h glucose levels for each 3-h increment in fasting duration (23). However, in that study, the overnight fasting duration was determined by a telephone 24 -h dietary recall conducted 3-10 days after the OGTT (23), which may have been associated with risk of recall bias. In our study, a $3 \mathrm{~h}$ longer fasting duration was associated with a 5.1\% higher 2-h glucose, which is substantial; that is, the 2-h glucose would increase from, for example, $6.4 \mathrm{mmol} / \mathrm{L}$ (median 2-h post-load glucose level) to $6.7 \mathrm{mmol} / \mathrm{L}$. There is a lack 
Table 2 Back transformed (from log scale) estimates in percent difference for associations between overnight fasting duration and glucose, insulin, glucagon, GIP and GLP-1.

\begin{tabular}{|c|c|c|c|c|c|c|c|c|}
\hline \multirow[b]{3}{*}{ Plasma glucos } & \multicolumn{4}{|c|}{ Unadjusted } & \multicolumn{4}{|c|}{ Adjusted for age, sex, BMI, smoking status and weekday } \\
\hline & \multirow[t]{2}{*}{$n$} & \multicolumn{2}{|c|}{ Estimate (\%) $95 \% \mathrm{Cl}$} & \multirow[t]{2}{*}{$P$} & \multirow[t]{2}{*}{$n$} & \multicolumn{2}{|c|}{ Estimate (\%) $95 \% \mathrm{Cl}$} & \multirow[t]{2}{*}{$P$} \\
\hline & & & & & & & & \\
\hline Fasting & 1717 & 0.0 & $(-0.4,0.3)$ & 0.844 & 1706 & -0.2 & $(-0.5,0.1)$ & 0.267 \\
\hline $30 \mathrm{~min}$ & 1702 & 0.4 & $(-0.2,0.9)$ & 0.159 & 1691 & 0.2 & $(-0.3,0.7)$ & 0.488 \\
\hline $120 \mathrm{~min}$ & 1710 & 2.0 & $(1.1,3.9)$ & $<0.001$ & 1699 & 1.7 & $(0.8,2.5)$ & $<0.001$ \\
\hline $\mathrm{rAUC}_{0-30}$ & 1702 & 0.2 & $(0.0,0.5)$ & 0.068 & 1691 & 0.2 & $(0.0,0.5)$ & 0.109 \\
\hline$r A \cup C_{0-120}$ & 1698 & 0.9 & $(0.5,1.3)$ & $<0.001$ & 1687 & 0.8 & $(0.4,1.2)$ & $<0.001$ \\
\hline \multicolumn{9}{|c|}{ Plasma insulin } \\
\hline Fasting & 1716 & 1.6 & $(-0.3,3.4)$ & 0.094 & 1705 & 0.7 & $(-0.9,2.2)$ & 0.400 \\
\hline $30 \mathrm{~min}$ & 1699 & 1.3 & $(-0.6,3.3)$ & 0.172 & 1688 & 0.7 & $(-1.2,2.5)$ & 0.481 \\
\hline $120 \mathrm{~min}$ & 1708 & 4.8 & $(2.3,7.2)$ & $<0.001$ & 1697 & 3.6 & $(1.4,5.8)$ & 0.001 \\
\hline $\mathrm{rAUC}_{0-30}$ & 1698 & 0.0 & $(-1.5,1.4)$ & 0.957 & 1687 & 0.2 & $(-1.2,1.6)$ & 0.805 \\
\hline rAUC $_{0-120}$ & 1694 & 1.2 & $(-0.2,2.7)$ & 0.084 & 1683 & 1.3 & $(-0.1,2.7)$ & 0.066 \\
\hline \multicolumn{9}{|c|}{ Plasma glucagon } \\
\hline Fasting & 1466 & 0.5 & $(-1.4,2.5)$ & 0.601 & 1455 & 0.3 & $(-1.8,1.9)$ & 0.967 \\
\hline $30 \mathrm{~min}$ & 1453 & 2.0 & $(0.0,4.0)$ & 0.048 & 1442 & 1.0 & $(-0.8,2.9)$ & 0.278 \\
\hline $120 \mathrm{~min}$ & 1457 & -0.4 & $(-3.0,2.3)$ & 0.783 & 1446 & -0.8 & $(-3.4,1.9)$ & 0.553 \\
\hline $\mathrm{rAUC}_{0-30}$ & 1450 & 0.2 & $(-0.5,1.0)$ & 0.579 & 1439 & 0.0 & $(-0.7,0.8)$ & 0.903 \\
\hline$r A \cup C_{0-120}$ & 1442 & 0.2 & $(-0.9,1.4)$ & 0.720 & 1431 & 0.0 & $(-1.1,1.2)$ & 0.972 \\
\hline \multicolumn{9}{|l|}{ Plasma GIP } \\
\hline Fasting & 1402 & -2.6 & $(-4.3,-0.9)$ & 0.003 & 1391 & -2.4 & $(-4.1,-0.7)$ & 0.006 \\
\hline $30 \mathrm{~min}$ & 1390 & 0.5 & $(-0.9,1.9)$ & 0.511 & 1380 & 0.5 & $(-0.9,1.9)$ & 0.468 \\
\hline $120 \mathrm{~min}$ & 1394 & 0.4 & $(-1.0,1.8)$ & 0.552 & 1383 & 0.7 & $(-0.7,2.0)$ & 0.328 \\
\hline $\mathrm{rAUC}_{0-30}$ & 1386 & 2.7 & $(1.2,4.3)$ & $<0.001$ & 1376 & 2.6 & $(1.1,4.1)$ & 0.001 \\
\hline$r A \cup C_{0-120}$ & 1377 & 3.0 & $(1.4,4.7)$ & $<0.001$ & 1367 & 3.0 & $(1.3,4.7)$ & 0.001 \\
\hline \multicolumn{9}{|c|}{ Plasma GLP-1 } \\
\hline Fasting & 1464 & -2.3 & $(-4.6,0.0)$ & 0.046 & 1453 & -2.9 & $(-5.1,-0.6)$ & 0.013 \\
\hline $30 \mathrm{~min}$ & 1453 & -1.5 & $(-2.5,1.5)$ & 0.601 & 1442 & -0.9 & $(-2.8,1.0)$ & 0.358 \\
\hline $120 \mathrm{~min}$ & 1457 & -0.8 & $(-2.4,0.9)$ & 0.380 & 1446 & -0.7 & $(-2.3,0.9)$ & 0.388 \\
\hline $\mathrm{rAUC}_{0-30}$ & 1448 & 1.4 & $(-0.5,3.2)$ & 0.149 & 1437 & 1.6 & $(-0.3,3.4)$ & 0.093 \\
\hline$r A \cup C_{0-120}$ & 1440 & 1.9 & $(-0.2,4.0)$ & 0.073 & 1429 & 2.3 & $(0.3,4.4)$ & 0.026 \\
\hline
\end{tabular}

Estimates are per $1 \mathrm{~h}$ increase in fasting duration. Linear regression models.

GIP glucose-dependent insulinotropic polypeptide; GLP-1, glucagon-like peptide 1; rAUC ${ }_{0-30}$, relative early response; rAUC ${ }_{0-120}$, relative total response.

of studies investigating associations of overnight fasting duration with glucagon, GIP and GLP-1, so our findings of lower GIP and GLP-1 levels in response to longer fasting duration are novel and need confirmation in other populations.

Participants examined on Mondays in the present study were slightly younger and had slightly lower BMI and $\mathrm{HbA}_{1 \mathrm{c}}$ than those examined on other weekdays, so these characteristics are unlikely to explain our findings of a slightly worse glucose regulation in those examined on Mondays. To our knowledge, no previous studies have investigated associations between weekday of examination and glucose levels during an OGTT. A study on triglyceride levels based on 1.8 million blood samples found 5\% higher levels on Mondays compared to Fridays among out-patients and 1.9\% among in-patients, suggesting that the effect of weekend behavior is likely to be attenuated when hospitalized (14). In a study of 807 Danish children with up to three different visit days, a marker of insulin resistance in the fasting state was found to be 35\% higher on Mondays compared to Fridays (15). These findings are in agreement with our observation of $20.6 \%$ higher fasting insulin levels and similar fasting glucose levels on Mondays. The higher fasting insulin levels on Mondays could possibly be due to 'weekend behavior', but we did not find higher glucose levels as we had also hypothesized.

No previous population-based studies have investigated associations between weekday of examination and GIP and GLP-1 levels. We did not find any differences in levels of GIP and GLP-1 on Mondays compared to the other days of the week. In general, fasting levels of gut hormones including GIP and GLP-1 are not very well defined, because the levels are usually close to the detection limit of the assays and likely to be influenced by non-specific factors, whereas the measured changes after glucose or meal ingestion are more likely to represent real changes in hormone concentration (24).

This work is licensed under a Creative Commons Attribution-NonCommercial-NoDerivatives 4.0 

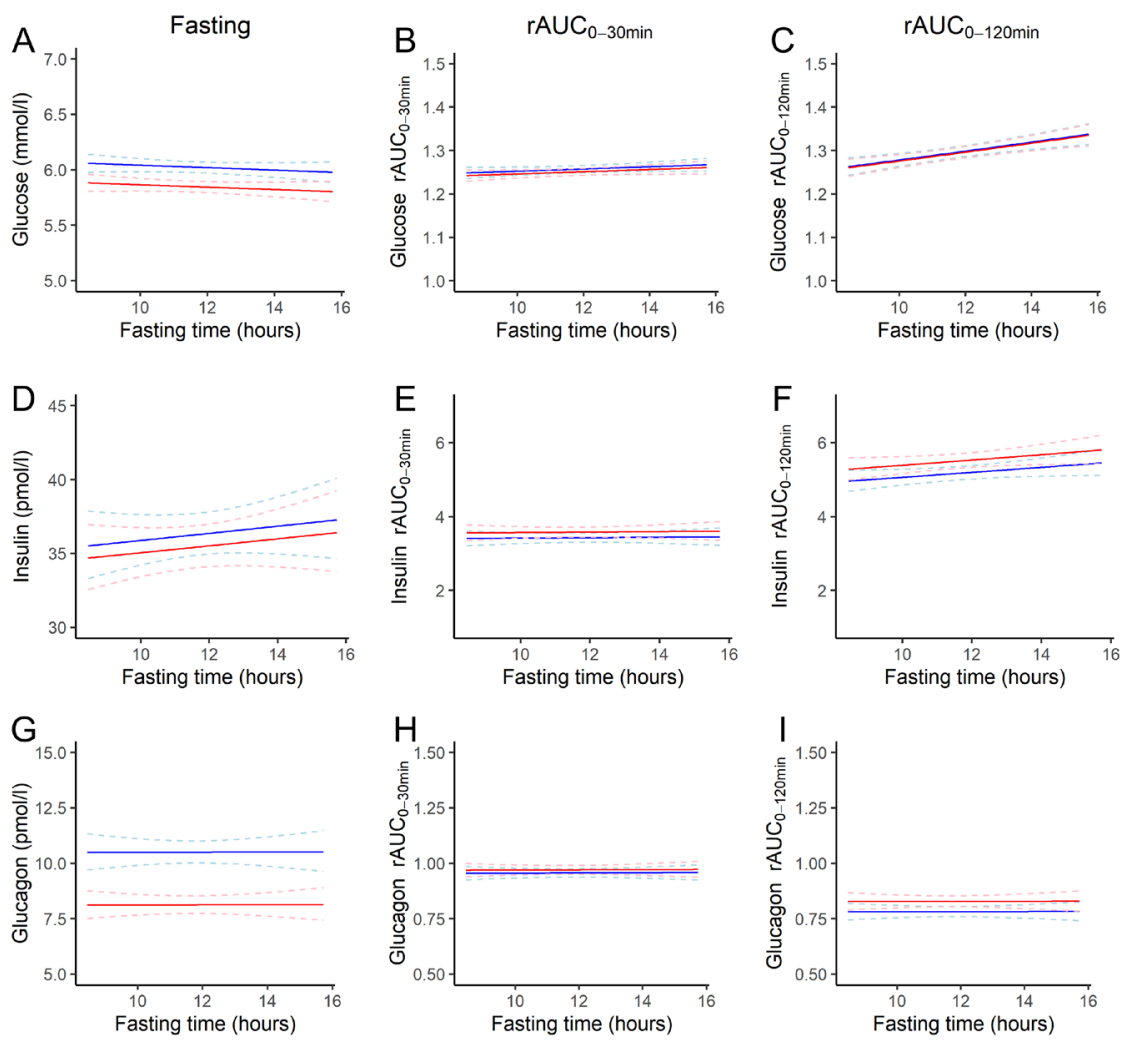

\section{Figure 1}

Relationship between fasting duration and fasting levels, $r A \cup C_{0-30}$ and $r A \cup C_{0-120}$ for glucose $(A, B$ and $C)$, insulin ( $D, E$ and $F$ ) and glucagon ( $G, H$ and I), respectively, for a hypothetical man (blue lines) and woman (red lines) aged 66 years (population mean), BMI $27 \mathrm{~kg} / \mathrm{m}^{2}$ (population mean), non-smoker (most frequent smoking status), not on a Monday. Dashed lines show the 95\% confidence intervals.

\section{Strengths and limitations}

We had a unique opportunity to assess associations of fasting duration and weekday of examination with different markers of glucose metabolism in the ADDITION-PRO cohort, which included more than 1700 individuals with data from standardized threepoint OGTTs with measurements of glucose, insulin and glucagon as well as the incretins GIP and GLP-1. A limitation of the study was that - as is standard for OGTT - the participants were instructed to fast for at least $8 \mathrm{~h}$ prior to the test. Hence, we were not able to investigate overnight fasting durations shorter than $8 \mathrm{~h}$. The fasting duration interquartile range only spanned a few hours in our study population (10.4-13.0 h) which limited our ability to conclude anything beyond this fasting
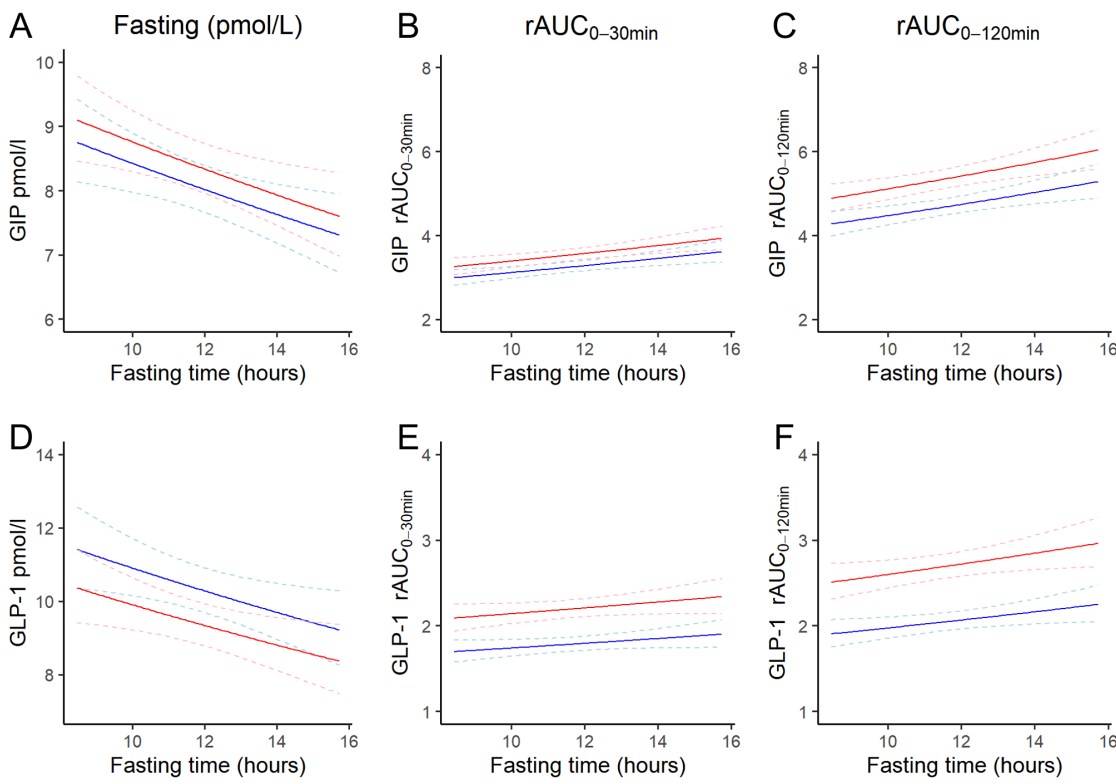

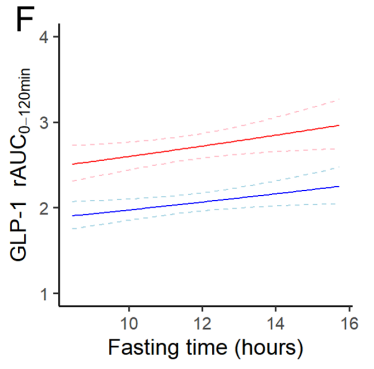

\section{Figure 2}

Relationship between fasting duration and fasting levels, $\mathrm{rAUC}_{0-30}$ and $\mathrm{rAUC}_{0-120}$ for GLP-1 (A, B and $C)$ and GIP (D, E and F), respectively, for a hypothetical man (blue lines) and woman (red lines) aged 66 years (population mean), BMI 27 $\mathrm{kg} / \mathrm{m}^{2}$ (population mean), non-smoker (most frequent smoking status), not on a Monday. Dashed lines show the $95 \%$ confidence intervals. https://ec.bioscientifica.com https://doi.org/10.1530/EC-20-0009 (c) 2020 The authors Published by Bioscientifica Ltd
This work is licensed under a Creative Commons Attribution-NonCommercial-NoDerivatives 4.0 denternationab bicense.ifica com at 04/26/2023 12:15:05PM 
Table 3 Back transformed (from log scale) estimates in percent difference between Mondays vs the other weekdays for glucose, insulin, glucagon, GIP and GLP-1.

\begin{tabular}{|c|c|c|c|c|c|c|c|c|}
\hline \multirow[b]{3}{*}{ Plasma glucos } & \multicolumn{4}{|c|}{ Unadjusted } & \multicolumn{4}{|c|}{$\begin{array}{c}\text { Adjusted for age, sex, BMI, smoking status and fasting } \\
\text { duration }\end{array}$} \\
\hline & \multirow[t]{2}{*}{$n$} & \multicolumn{2}{|c|}{ Estimate (\%) $95 \% \mathrm{Cl}$} & \multirow[t]{2}{*}{$P$} & \multirow[t]{2}{*}{$n$} & \multicolumn{2}{|c|}{ Estimate (\%) 95\% Cl } & \multirow[t]{2}{*}{$P$} \\
\hline & & & & & & & & \\
\hline Fasting & 1718 & 0.9 & $(-0.9,2.7)$ & 0.316 & 1706 & 1.7 & $(0.0,3.4)$ & 0.051 \\
\hline $30 \mathrm{~min}$ & 1703 & -2.1 & $(-4.8,0.7)$ & 0.134 & 1691 & -0.9 & $(-3.5,1.8)$ & 0.508 \\
\hline $120 \mathrm{~min}$ & 1711 & -5.1 & $(-9.5,-0.6)$ & 0.028 & 1699 & -2.9 & $(-7.2,1.5)$ & 0.187 \\
\hline $\mathrm{rAUC}_{0-30}$ & 1703 & -1.8 & $(-3.1,-0.5)$ & 0.007 & 1691 & -1.5 & $(-2.8,-0.2)$ & 0.020 \\
\hline$r^{\prime} A \cup C_{0-120}$ & 1699 & -3.6 & $(-5.6,-1.6)$ & $<0.001$ & 1687 & -2.9 & $(-4.8,-0.9)$ & 0.004 \\
\hline \multicolumn{9}{|c|}{ Plasma insulin } \\
\hline Fasting & 1717 & 14.8 & $(4.4,26.3)$ & 0.005 & 1705 & 20.6 & $(11.2,30.7)$ & $<0.001$ \\
\hline $30 \mathrm{~min}$ & 1700 & 8.7 & $(-1.7,20.1)$ & 0.104 & 1688 & 13.1 & $(2.8,24.4)$ & 0.012 \\
\hline $120 \mathrm{~min}$ & 1709 & 1.8 & $(-9.9,15.1)$ & 0.774 & 1697 & 9.1 & $(-2.5,22.1)$ & 0.128 \\
\hline $\mathrm{rAUC}_{0-30}$ & 1699 & -5.5 & $(-12.4,2.0)$ & 0.145 & 1687 & -6.4 & $(-13.1,0.9)$ & 0.084 \\
\hline $\mathrm{rAUC}_{0-120}$ & 1695 & -8.4 & $(-14.9,-1.5)$ & 0.019 & 1683 & -8.4 & $(-14.7,-1.5)$ & 0.017 \\
\hline \multicolumn{9}{|c|}{ Plasma glucagon } \\
\hline Fasting & 1467 & 3.8 & $(-5.7,14.2)$ & 0.445 & 1455 & 6.1 & $(-3.2,16.2)$ & 0.207 \\
\hline $30 \mathrm{~min}$ & 1454 & -0.8 & $(-10.1,9.3)$ & 0.864 & 1442 & 3.1 & $(-6.0,13.1)$ & 0.514 \\
\hline $120 \mathrm{~min}$ & 1458 & 2.7 & $(-9.9,17.0)$ & 0.693 & 1446 & 5.7 & $(-7.2,20.5)$ & 0.405 \\
\hline $\mathrm{rAUC}_{0-30}$ & 1451 & -2.7 & $(-6.2,1.1)$ & 0.158 & 1439 & -2.0 & $(-5.6,1.8)$ & 0.299 \\
\hline$r A \cup C_{0-120}$ & 1443 & -1.8 & $(-7.2,3.9)$ & 0.537 & 1431 & -0.9 & $(-6.3,4.9)$ & 0.765 \\
\hline \multicolumn{9}{|l|}{ Plasma GIP } \\
\hline Fasting & 1403 & -1.3 & $(-9.5,7.8)$ & 0.777 & 1391 & -2.1 & $(-10.3,6.8)$ & 0.626 \\
\hline $30 \mathrm{~min}$ & 1391 & 3.4 & $(-3.7,11.0)$ & 0.354 & 1380 & 4.0 & $(-3.0,11.6)$ & 0.270 \\
\hline $120 \mathrm{~min}$ & 1395 & 3.8 & $(-3.1,11.2)$ & 0.292 & 1383 & 3.6 & $(-3.0,10.7)$ & 0.296 \\
\hline rAUC $_{0-30}$ & 1387 & 4.7 & $(-2.7,12.7)$ & 0.221 & 1376 & 5.8 & $(-1.6,13.9)$ & 0.129 \\
\hline rAUC $_{-120}$ & 1378 & 5.1 & $(-3.1,14.1)$ & 0.228 & 1367 & 6.3 & $(-2.0,15.2)$ & 0.139 \\
\hline \multicolumn{9}{|c|}{ Plasma GLP-1 } \\
\hline Fasting & 1465 & -7.4 & $(-17.4,3.9)$ & 0.193 & 1453 & -3.7 & $(-14.1,7.9)$ & 0.518 \\
\hline $30 \mathrm{~min}$ & 1454 & -2.3 & $(-11.6,7.9)$ & 0.646 & 1442 & 1.1 & $(-8.2,11.4)$ & 0.826 \\
\hline $120 \mathrm{~min}$ & 1458 & 0.5 & $(-7.6,9.3)$ & 0.911 & 1446 & 2.2 & $(-5.6,10.6)$ & 0.595 \\
\hline $\mathrm{rAUC}_{0-30}$ & 1449 & 3.4 & $(-5.5,13.2)$ & 0.464 & 1437 & 2.8 & $(-6.0,12.4)$ & 0.549 \\
\hline$r A \cup C_{0-120}$ & 1441 & 6.2 & $(-4.0,17.6)$ & 0.243 & 1429 & 4.7 & $(-5.1,15.6)$ & 0.359 \\
\hline
\end{tabular}

Linear regression models.

GIP glucose-dependent insulinotropic polypeptide; GLP-1, and glucagon-like peptide 1; rAUC $\mathrm{C}_{0-30}$, relative early response; rAUC $\mathrm{C}_{0-120}$, relative total response.

duration. However, this is likely to reflect real-life lengths of overnight fasting (12), and we believe the results are both generalizable and relevant to real-life situations. As this is a cross-sectional study, we do not know whether the fasting duration on the day of examination reflects the habitual overnight fasting durations. The study population was sampled specifically to include both individuals with low as well as high risk of diabetes; this was done to ensure that participants with different levels of diabetes risk were included. It consisted only of middle-aged and older individuals, and only half of the invited individuals participated. The attendees were more likely to be men, overweight and to have a family history of diabetes (16). We cannot rule out that there are interindividual differences in the participants that attended ADDITION-PRO on different days of the week, which was not explained by the confounders adjusted for in the analysis. Nevertheless, differences in clinical characteristics are not likely to explain the findings; if anything, the health status of those attending examination on a Monday was slightly better than those examined on the other weekdays. The reason for this remains unknown.

A previous study found that the mean age in those with a social jetlag of $<1 \mathrm{~h}$ was 64 years compared to 57 years in those with a social jetlag $\geq 1 \mathrm{~h}$ (25). Also, the increase in total energy intake from weekdays to the weekends seems to be more pronounced among those aged 18-64 years compared to those aged $\geq 65$ years (13). Accordingly, the relatively high mean age of the participants in the present study might translate into less pronounced differences between weekday and weekend behavior than would be expected in a younger population. https://ec.bioscientifica.com https://doi.org/10.1530/EC-20-0009 (c) 2020 The authors Published by Bioscientifica Ltd

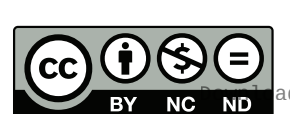

This work is licensed under a Creative Commons Attribution-NonCommercial-NoDerivatives 4.0 Internationab bicense.ifica .com at 04/26/2023 12:15:05PM 


\section{Implications}

The basis for prevention of type 2 diabetes among overweight individuals at high risk of developing the disease is weight loss and increased physical activity (26). Since maintaining the initial weight-loss after participating in a weight loss program is difficult (27), it is important to have multiple tools at one's disposal when recommending lifestyle changes to individuals at high risk of diabetes. Restricting the time of eating during the day to a shorter time interval has shown potential as a weight loss strategy (12). Restricting the daily eating window (so-called 'time-restricted eating') has been shown to lead to reductions in energy intake $(12,28,29)$. Despite this fact, a randomized cross-over study found that insulin sensitivity was improved in response to 'early time-restricted eating' (eating duration: $6 \mathrm{~h}$ /day, dinner before 15:00 h) despite no reduction in energy intake and body weight (30). The study found no differences in glucose concentrations during an OGTT between the groups (30). However, recent short-term cross-over studies have suggested that 'early time-restricted eating' with concomitant longer fasting duration is associated with improved glucose tolerance during a mixed meal test and reductions in fasting glucose concentrations (31), as well as in 24-h mean glucose and glycaemic variability (32) during continuous glucose monitoring despite similar energy intake (32). As we did not see lower fasting plasma glucose levels among those with longer fasting duration, this can also be used to reassure people that their body can maintain a normal blood glucose level even after having a longer overnight fast. Late night food consumption has been proposed as a risk factor for type 2 diabetes and cardiometabolic health (33), which may possibly be explained by a shorter overnight fasting duration and excess energy intake. Also, experimental circadian misalignment has been shown to lead to decreased glucose tolerance $(34,35)$. Less pronounced cases of circadian misalignment could be the result of shorter overnight fasting durations.

The present study points to an association between weekday of examination and glucose metabolism. This finding may be due to different weekend behavior, for example, different sleeping and eating patterns, compared to weekdays (25). Therefore, examining the participants on the same day of the week in studies with repeated measurements seems prudent when logistically possible; however, studies with randomised day of examination are needed to confirm the results.

\section{Conclusion}

We found that longer fasting duration is associated with slightly higher 2-h glucose and insulin levels and greater GIP and GLP-1 response after glucose ingestion. Additionally, we found higher fasting insulin levels and similar fasting glucose levels on Mondays compared to the other days of the week pointing to a worse glucose regulation after the weekend.

\section{Declaration of interest}

K K B C, J S Q, D V, M E J and K F are employed by Steno Diabetes Center Copenhagen, a research hospital working in the Danish National Health Service. Until 31 December 2016 Steno Diabetes Center was owned by Novo Nordisk A/S and received part of its core funding from unrestricted grants from the Novo Nordisk Foundation and Novo Nordisk A/S. K K B C, K F, D V and M E J own shares in Novo Nordisk A/S. The other authors have nothing to disclose.

\section{Funding}

The ADDITION-Denmark study was funded by the National Health Services in the counties of Copenhagen, Aarhus, Ringkøbing, Ribe, and Southern Jutland in Denmark; the Danish Council for Strategic Research; the Danish Research Foundation for General Practice; Novo Nordisk Foundation; the Danish Centre for Evaluation and Health Technology Assessment; the Diabetes Fund of the National Board of Health; the Danish Medical Research Council and the Aarhus University Research Foundation. The ADDITION-PRO study was funded by an unrestricted grant from the European Foundation for the Study of Diabetes/Pfizer for Research into cardiovascular disease risk reduction in patients with diabetes (74550801), the Danish Council for Strategic Research and internal research and equipment funds from Steno Diabetes Center. Between 2000 and 2011, the ADDITION study (screening and intensive treatment of type 2 diabetes in primary care) received unrestricted grants from the medical industry: Novo Nordisk AS, Novo Nordisk Scandinavia AB, ASTRA Denmark, Pfizer Denmark, GlaxoSmithKline Pharma Denmark, SERVIER Denmark A/S and HemoCue Denmark A/S.

\section{Author contribution statement}

K K B C drafted the manuscript and performed the statistical analysis with support from D V. K F, D V and K K B C developed the analysis plan. The ADDITION-PRO study was designed by D R W. J J H and S T provided the glucagon, GIP and GLP-1 data. All authors contributed to interpretation of data, revised the manuscript critically and have read and approved the final version of the manuscript.

\section{Acknowledgements}

Without the work and contributions by the ADDITION-PRO study centres, the staff and the participants, this work would not have been possible.

\section{References}

1 Van Cauter E, Polonsky KS \& Scheen AJ. Roles of circadian rhythmicity and sleep in human glucose regulation. Endocrine Reviews 199718 716-738. (https://doi.org/10.1210/edrv.18.5.0317)

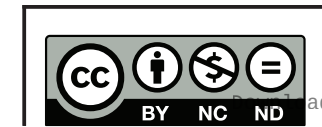

This work is licensed under a Creative Commons Attribution-NonCommercial-NoDerivatives 4.0 enternationad ticense ifica.com at 04/26/2023 12:15:05PM 
2 Zimmet PZ, Wall JR, Rome R, Stimmler L \& Jarrett RJ. Diurnal variation in glucose tolerance: associated changes in plasma insulin, growth hormone, and non-esterified fatty acids. BMJ 19741 485-488. (https://doi.org/10.1136/bmj.1.5906.485)

3 Hulmán A, Færch K, Vistisen D, Karsai J, Nyári TA, Tabák AG, Brunner EJ, Kivimäki M \& Witte DR. Effect of time of day and fasting duration on measures of glycaemia: analysis from the Whitehall II study. Diabetologia 201356 294-297. (https://doi.org/10.1007/ s00125-012-2770-3)

4 Baggio LL \& Drucker DJ. Biology of incretins: GLP-1 and GIP. Gastroenterology 2007132 2131-2157. (https://doi.org/10.1053/j. gastro.2007.03.054)

5 Holst JJ, Knop FK, Vilsbøll T, Krarup T \& Madsbad S. Loss of incretin effect is a specific, important, and early characteristic of type 2 diabetes. Diabetes Care 201134 (Supplement 2) S251-S257. (https:// doi.org/10.2337/dc11-s227)

6 Drucker DJ. The biology of incretin hormones. Cell Metabolism 2006 3 153-165. (https://doi.org/10.1016/j.cmet.2006.01.004)

7 Vilsbøll T \& Holst JJ. Incretins, insulin secretion and type 2 diabetes mellitus. Diabetologia 200447 357-366. (https://doi.org/10.1007/ s00125-004-1342-6)

8 Muscelli E, Mari A, Casolaro A, Camastra S, Seghieri G, Gastaldelli A, Holst JJ \& Ferrannini E. Separate impact of obesity and glucose tolerance on the incretin effect in normal subjects and type 2 diabetic patients. Diabetes 200857 1340-1348. (https://doi. org/10.2337/db07-1315)

9 Mingrone G, Nolfe G, Castagneto Gissey GC, Iaconelli A, Leccesi L, Guidone C, Nanni G \& Holst JJ. Circadian rhythms of GIP and GLP1 in glucose-tolerant and in type 2 diabetic patients after biliopancreatic diversion. Diabetologia 200952 873-881. (https://doi. org/10.1007/s00125-009-1288-9)

10 Galindo Muñoz JS, Jiménez Rodríguez D \& Hernández Morante JJ. Diurnal rhythms of plasma GLP-1 levels in normal and overweight/ obese subjects: lack of effect of weight loss. Journal of Physiology and Biochemistry 201571 17-28. (https://doi.org/10.1007/s13105-0140375-7)

11 Wittmann M, Dinich J, Merrow M \& Roenneberg T. Social jetlag: misalignment of biological and social time. Chronobiology International 200623 497-509. (https://doi. org/10.1080/07420520500545979)

12 Gill S \& Panda S. A smartphone app reveals erratic diurnal eating patterns in humans that can be modulated for health benefits. Cell Metabolism 201522 789-798. (https://doi.org/10.1016/j. cmet.2015.09.005)

13 An R. Weekend-weekday differences in diet among U.S. adults, 2003-2012. Annals of Epidemiology 201626 57-65. (https://doi. org/10.1016/j.annepidem.2015.10.010)

14 Jaskolowski J, Ritz C, Sjödin A, Astrup A, Szecsi PB, Stender S \& Hjorth MF. Weekday variation in triglyceride concentrations in 1.8 million blood samples. Journal of Lipid Research 201758 1204-1213. (https://doi.org/10.1194/jlr.M074062)

15 Hjorth MF, Damsgaard CT, Michaelsen KF, Astrup A \& Sjödin A. Markers of metabolic health in children differ between weekdays-the result of unhealthier weekend behavior. Obesity 201523 733-736. (https://doi.org/10.1002/oby.21034)

16 Johansen NB, Hansen AL, Jensen TM, Philipsen A, Rasmussen SS, Jørgensen ME, Simmons RK, Lauritzen T, Sandbæk A \& Witte DR Protocol for ADDITION-PRO: a longitudinal cohort study of the cardiovascular experience of individuals at high risk for diabetes recruited from Danish primary care. BMC Public Health 201212 1078. (https://doi.org/10.1186/1471-2458-12-1078)

17 Glümer C, Carstensen B, Sandbaek A, Lauritzen T, Jørgensen T, Borch-Johnsen K \& Inter99 Study. A Danish diabetes risk score for targeted screening: the Inter99 study. Diabetes Care 200427 727-733. (https://doi.org/10.2337/diacare.27.3.727)
18 Færch K, Torekov SS, Vistisen D, Johansen NB, Witte DR, Jonsson A, Pedersen O, Hansen T, Lauritzen T, Sandbæk A, et al. GLP-1 response to oral glucose is reduced in prediabetes, screen-detected type 2 diabetes, and obesity and influenced by sex: the ADDITION-PRO study. Diabetes 201564 2513-2525. (https://doi.org/10.2337/db141751)

19 Færch K, Vistisen D, Pacini G, Torekov SS, Johansen NB, Witte DR, Jonsson A, Pedersen $\mathrm{O}$, Hansen T, Lauritzen T, et al. Insulin resistance is accompanied by increased fasting glucagon and delayed glucagon suppression in individuals with normal and impaired glucose regulation. Diabetes 201665 3473-3481. (https://doi.org/10.2337/ db16-0240)

20 Møller CL, Vistisen D, Færch K, Johansen NB, Witte DR, Jonsson A, Pedersen O, Hansen T, Lauritzen T, Jørgensen ME, et al. Glucosedependent insulinotropic polypeptide is associated with lower low-density lipoprotein but unhealthy fat distribution, independent of insulin: the ADDITION-PRO study. Journal of Clinical Endocrinology and Metabolism 2016101 485-493. (https://doi.org/10.1210/jc.20153133)

21 Jørgensen SW, Brøns C, Bluck L, Hjort L, Faerch K, Thankamony A, Gillberg L, Friedrichsen M, Dunger DB \& Vaag AA. Metabolic response to 36 hours of fasting in young men born small vs appropriate for gestational age. Diabetologia 201558 178-187. (https://doi.org/10.1007/s00125-014-3406-6)

22 Moebus S, Göres L, Lösch C \& Jöckel KH. Impact of time since last caloric intake on blood glucose levels. European Journal of Epidemiology 201126 719-728. (https://doi.org/10.1007/s10654-0119608-z)

23 Marinac CR, Natarajan L, Sears DD, Gallo LC, Hartman SJ, Arredondo E \& Patterson RE. Prolonged nightly fasting and breast cancer risk: findings from NHANES (2009-2010). Cancer Epidemiology, Biomarkers and Prevention 201524 783-789. (https:// doi.org/10.1158/1055-9965.EPI-14-1292)

24 Deacon CF \& Holst JJ. Immunoassays for the incretin hormones GIP and GLP-1. Best Practice and Research: Clinical Endocrinology and Metabolism 200923 425-432. (https://doi.org/10.1016/j. beem.2009.03.006)

25 Koopman ADM, Rauh SP, Van 't Riet E, Groeneveld L, Van Der Heijden AA, Elders PJ, Dekker JM, Nijpels G, Beulens JW \& Rutters F. The association between social jetlag, the metabolic syndrome, and type 2 diabetes mellitus in the general population: the new Hoorn study. Journal of Biological Rhythms 201732 359-368. (https://doi. org/10.1177/0748730417713572)

26 World Health Organization. Diet, nutrition and the prevention of chronic diseases: Report of the joint WHO/FAO expert consultation. Geneva, Switzerland: WHO, 2003. (available at: https://www.who.int/ dietphysicalactivity/publications/trs916/en/)

27 Anderson JW, Konz EC, Frederich RC \& Wood CL. Long-term weightloss maintenance: a meta-analysis of US studies. American Journal of Clinical Nutrition $2001 \mathbf{7 4} 579-584$. (https://doi.org/10.1093/ ajcn/74.5.579)

28 Gabel K, Hoddy KK, Haggerty N, Song J, Kroeger CM, Trepanowski JF, Panda S \& Varady KA. Effects of 8-hour time restricted feeding on body weight and metabolic disease risk factors in obese adults: a pilot study. Nutrition and Healthy Aging 20184 345-353. (https://doi. org/10.3233/NHA-170036)

29 Wilkinson MJ, Manoogian ENC, Zadourian A, Lo H, Fakhouri S, Shoghi A, Wang X, Fleischer JG, Navlakha S, Panda S, et al. Ten-hour time-restricted eating reduces weight, blood pressure, and atherogenic lipids in patients with metabolic syndrome. Cell Metabolism 202031 92-104.e5. (https://doi.org/10.1016/j. cmet.2019.11.004)

30 Sutton EF, Beyl R, Early KS, Cefalu WT, Ravussin E \& Peterson CM. Early time-restricted feeding improves insulin sensitivity, blood pressure, and oxidative stress even without weight loss in men with https://ec.bioscientifica.com https://doi.org/10.1530/EC-20-0009 (c) 2020 The authors Published by Bioscientifica Ltd
This work is licensed under a Creative Commons Attribution-NonCommercial-NoDerivatives 4.0 Internationab License.ifica com at $04 / 26 / 2023$ 12:15:05PM 
prediabetes. Cell Metabolism 201827 1212-1221.e3. (https://doi. org/10.1016/j.cmet.2018.04.010)

31 Hutchison AT, Regmi P, Manoogian ENC, Fleischer JG, Wittert GA, Panda S \& Heilbronn LK. Time-restricted feeding improves glucose tolerance in men at risk for type 2 diabetes: a randomized crossover trial. Obesity 201927 724-732. (https://doi.org/10.1002/oby.22449)

32 Jamshed H, Beyl RA, Della Manna DL, Yang ES, Ravussin E \& Peterson CM. Early time-restricted feeding improves 24-hour glucose levels and affects markers of the circadian clock, aging, and autophagy in humans. Nutrients 201911 1234. (https://doi. org/10.3390/nu11061234)

33 St-Onge MP, Ard J, Baskin ML, Chiuve SE, Johnson HM, KrisEtherton P, Varady K \& American Heart Association Obesity
Committee of the Council on Lifestyle and Cardiometabolic Health; Council on Cardiovascular Disease in the Young; Council on Clinical Cardiology; and Stroke Council. Meal timing and frequency: implications for cardiovascular disease prevention a scientific statement from the American Heart Association. Circulation 2017 135 e96-e121. (https://doi.org/10.1161/CIR.0000000000000476) 34 Stenvers DJ, Scheer FAJL, Schrauwen P, la Fleur SE \& Kalsbeek A. Circadian clocks and insulin resistance. Nature Reviews: Endocrinology 201915 75-89. (https://doi.org/10.1038/s41574-018-0122-1)

35 Qian J \& Scheer FAJL. Circadian system and glucose metabolism: implications for physiology and disease. Trends in Endocrinology and Metabolism 201627 282-293. (https://doi.org/10.1016/j. tem.2016.03.005)

Received in final form 19 February 2020

Accepted 11 March 2020

Accepted Manuscript published online 11 March 2020 (c) 2020 The authors Published by Bioscientifica Ltd 